\title{
A Convolution Approach on Partial Sums of Certain Harmonic Univalent Functions
}

\author{
Saurabh Porwal \\ Department of Mathematics, UIET Campus, CSJM University, Kanpur 208024, India \\ Correspondence should be addressed to Saurabh Porwal, saurabhjcb@rediffmail.com
}

Received 30 March 2012; Revised 13 October 2012; Accepted 14 October 2012

Academic Editor: S. S. Dragomir

Copyright (C 2012 Saurabh Porwal. This is an open access article distributed under the Creative Commons Attribution License, which permits unrestricted use, distribution, and reproduction in any medium, provided the original work is properly cited.

The purpose of the present paper is to establish some new results giving the sharp bounds of the real parts of ratios of harmonic univalent functions to their sequences of partial sums by using convolution. Relevant connections of the results presented here with various known results are briefly indicated.

\section{Introduction}

A continuous complex-valued function $f=u+i v$ is said to be harmonic in a simply connected domain $D$ if both $u$ and $v$ are real harmonic in $D$. In any simply-connected domain we can write $f=h+\bar{g}$, where $h$ and $g$ are analytic in $D$. We call $h$ the analytic part and $g$ the co-analytic part of $f$. A necessary and sufficient condition for $f$ to be locally univalent and sense-preserving in $D$ is that $\left|h^{\prime}(z)\right|>\left|g^{\prime}(z)\right|, z \in D$, see [1]. For more basic results on harmonic functions one may refer to the following standard text book by Duren [2]. See also Ahuja [3] and Ponnusamy and Rasila ([4, $5])$.

Denote by $S_{H}$ the class of functions $f=h+\bar{g}$ which are harmonic univalent and sensepreserving in the open unit disk $U=\{z:|z|<1\}$ for which $f(0)=f_{z}(0)-1=0$. Then for $f=h+\bar{g} \in S_{H}$ we may express the analytic functions $h$ and $g$ as

$$
h(z)=z+\sum_{k=2}^{\infty} a_{k} z^{k}, \quad g(z)=\sum_{k=1}^{\infty} b_{k} z^{k}, \quad\left|b_{1}\right|<1 .
$$


Note that $S_{H}$ reduces to the class $S$ of normalized analytic univalent functions, if the coanalytic part of its member is zero, that is, $g \equiv 0$, and for this class $f(z)$ may be expressed as

$$
f(z)=z+\sum_{k=2}^{\infty} a_{k} z^{k}
$$

Let $\phi(z) \in S_{H}$ be a fixed function of the form

$$
\phi(z)=z+\sum_{k=2}^{\infty} c_{k} z^{k}+\overline{\sum_{k=1}^{\infty} d_{k} z^{k}}, \quad\left(d_{k} \geq c_{k} \geq c_{2}>0 ; k \geq 2,\left|d_{1}\right|<1\right) .
$$

Now, we introduce a class $S_{H}\left(c_{k}, d_{k}, \delta\right)$ consisting of functions of the form (1.1) which satisfies the inequality

$$
\sum_{k=2}^{\infty} c_{k}\left|a_{k}\right|+\sum_{k=1}^{\infty} d_{k}\left|b_{k}\right| \leq \delta, \quad \text { where } \delta>0
$$

and we note that if $d_{k}=0$, then the class $S_{H}\left(c_{k}, d_{k}, \delta\right)$ reduces to the class $S_{\phi}\left(c_{k}, \delta\right)$ which was introduced by Frasin [6]. In this case the condition (1.4) reduces to

$$
\sum_{k=2}^{\infty} c_{k}\left|a_{k}\right| \leq \delta, \quad \text { where } \delta>0
$$

It is easy to see that various subclasses of $S_{H}$ consisting of functions $f(z)$ of the form (1.1) can be represented as $S_{H}\left(c_{k}, d_{k}, \delta\right)$ for suitable choices of $c_{k}, d_{k}$, and $\delta$ studied earlier by various researchers. For example:

(1) $S_{H}(k, k, 1) \equiv S_{H}^{*}$ and $S_{H}\left(k^{2}, k^{2}, 1\right) \equiv K_{H}$ studied by Silverman [7]; Silverman and Silvia [8].

(2) $S_{H}(k-\alpha, k+\alpha, 1-\alpha) \equiv S_{H}^{*}(\alpha)$ studied by Jahangiri [9].

(3) $S_{H}((k-\alpha)(\phi(k, \lambda)),(k+\alpha)(\phi(k, \lambda)), 1-\alpha) \equiv S_{H, \lambda}^{*}(\alpha)$ studied by Dixit and Porwal [10].

(4) $S_{H}\left(k^{m}-\alpha k^{n}, k^{m}-\alpha k^{n}, 1-\alpha\right) \equiv H S(m, n, \alpha)$ studied by Dixit and Porwal [11].

(5) $S_{H}(k, k, \beta-1) \equiv H P(\beta)$ studied by Dixit and Porwal [12].

(6) $S_{H}\left(\lambda_{k}(1-\alpha \lambda)-\alpha(1-\lambda), \mu_{k}(1-\alpha \lambda)+\alpha(1-\lambda), 1-\alpha\right) \equiv S_{H}(\Phi, \Psi, \alpha, \lambda)$ studied by Dixit and Porwal [13].

(7) $S_{H}\left(\lambda_{k}-\alpha, \mu_{k}+\alpha, 1-\alpha\right) \equiv S_{H}(\Phi, \Psi, \alpha)$ studied by Frasin [14].

(8) $S_{H}(k(1-\alpha \lambda)-\alpha(1-\lambda), k(1-\alpha \lambda)+\alpha(1-\lambda), 1-\alpha) \equiv S_{H}^{*}(\alpha, \lambda)$ studied by Öztürk et al. [15].

(9) $\left.S_{H}\left((k(\beta+1)-t(\beta+\gamma)) \Gamma\left(\alpha_{1}, k\right),(k(\beta+1)+t(\beta+\gamma)) \Gamma\left(\alpha_{1}, k\right)\right),(k-1) !(1-\gamma)\right) \equiv$ $G_{H}\left(\alpha_{1}, \beta, \gamma, t\right)$ studied by Porwal et al. [16].

(10) $S_{H}(2 k-1-\alpha, 2 k+1+\alpha, 1-\alpha) \equiv G_{H}(\alpha)$ studied by Rosy et al. [17]. 
In 1985, Silvia [18] studied the partial sums of convex functions of order $\alpha$. Later on, Silverman [19], Afaf et al. [20], Dixit and Porwal [21], Frasin ([6, 22]), Murugusundaramoorthy et al. [23], Owa et al. [24], Porwal and Dixit [25], Raina and Bansal [26] and Rosy et al. [27] studied and generalized the results on partial sums for various classes of analytic functions. Very recently, Porwal [28], Porwal and Dixit [29] studied analogues interesting results on the partial sums of certain harmonic univalent functions. In this work, we extend all these results.

Now, we let the sequences of partial sums of function of the form (1.1) with $b_{1}=0$ be

$$
\begin{aligned}
& f_{m}(z)=z+\sum_{k=2}^{m} a_{k} z^{k}+\sum_{k=2}^{\infty} \overline{b_{k} z^{k}} \\
& f_{n}(z)=z+\sum_{k=2}^{\infty} a_{k} z^{k}+\sum_{k=2}^{n} \overline{b_{k} z^{k}} \\
& f_{m, n}(z)=z+\sum_{k=2}^{m} a_{k} z^{k}+\sum_{k=2}^{n} \overline{b_{k} z^{k}}
\end{aligned}
$$

when the coefficients of $f$ are sufficiently small to satisfy the condition (1.4).

In the present paper, we determine sharp lower bounds for $\operatorname{Re}\left\{(f(z) * \psi(z)) /\left(f_{m}(z) *\right.\right.$ $\psi(z))\}, \operatorname{Re}\left\{\left(f_{m}(z) * \psi(z)\right) /(f(z) * \psi(z))\right\}, \operatorname{Re}\left\{(f(z) * \psi(z)) /\left(f_{n}(z) * \psi(z)\right)\right\}, \operatorname{Re}\left\{\left(f_{n}(z) *\right.\right.$ $\psi(z)) /(f(z) * \psi(z))\}, \operatorname{Re}\left\{(f(z) * \psi(z)) /\left(f_{m, n}(z) * \psi(z)\right)\right\}$, and $\operatorname{Re}\left\{\left(f_{m, n}(z) * \psi(z)\right) /(f(z) *\right.$ $\psi(z))\}$ where $f_{m}(z), f_{n}(z)$ and $f_{m, n}(z)$ are defined above and $\psi(z)=z+\sum_{k=2}^{\infty} \lambda_{k} z^{k}+\sum_{k=2}^{\infty} \overline{\mu_{k} z^{k}}$, $\left(\lambda_{k} \geq 0, \mu_{k} \geq 0\right)$ is a harmonic function and the operator " * $*$ " stands for the Hadamard product or convolution of two power series, which is defined for two functions $f(z)$ and $g(z)$ are of the form

$$
\begin{aligned}
& f(z)=z+\sum_{k=2}^{\infty} a_{k} z^{k}+\sum_{k=2}^{\infty} \overline{b_{k} z^{k}}, \\
& g(z)=z+\sum_{k=2}^{\infty} c_{k} z^{k}+\sum_{k=2}^{\infty} \overline{d_{k} z^{k}}
\end{aligned}
$$

as

$$
(f * g)(z)=f(z) * g(z)=z+\sum_{k=2}^{\infty} a_{k} c_{k} z^{k}+\sum_{k=2}^{\infty} \overline{b_{k} d_{k} z^{k}}
$$

It is worthy to note that this study not only gives as a particular case, the results of Porwal [28], Porwal and Dixit [29], but also give rise to several new results.

\section{Main Results}

In our first theorem, we determine sharp lower bounds for $\operatorname{Re}\left\{(f(z) * \psi(z)) /\left(f_{m}(z) * \psi(z)\right)\right\}$. 
Theorem 2.1. If $f$ of the form (1.1) with $b_{1}=0$, satisfies the condition (1.4), then

$$
\operatorname{Re}\left\{\frac{f(z) * \psi(z)}{f_{m}(z) * \psi(z)}\right\} \geq \frac{c_{m+1}-\lambda_{m+1} \delta}{c_{m+1}}, \quad(z \in U)
$$

where

$$
c_{k} \geq \begin{cases}\lambda_{k} \delta & \text { if } k=2,3, \ldots, m \\ \frac{\lambda_{k} c_{m+1}}{\lambda_{m+1}} & \text { if } k=m+1, m+2 \ldots\end{cases}
$$

The result (2.1) is sharp with the function given by

$$
f(z)=z+\frac{\delta}{c_{m+1}} z^{m+1}
$$

where $0<\delta \leq c_{m+1} / \lambda_{m+1}$.

Proof. To obtain sharp lower bound given by (2.1), let us put

$$
\begin{aligned}
& \frac{1+\omega(z)}{1-\omega(z)} \\
& =\frac{c_{m+1}}{\lambda_{m+1} \delta}\left[\frac{f\left(r e^{i \theta}\right) * \psi\left(r e^{i \theta}\right)}{f_{m}\left(r e^{i \theta}\right) * \psi\left(r e^{i \theta}\right)}-\frac{c_{m+1}-\lambda_{m+1} \delta}{c_{m+1}}\right] \\
& =\frac{1+\sum_{k=2}^{m} \lambda_{k} a_{k} r^{k-1} e^{i(k-1) \theta}+\sum_{k=2}^{\infty} \mu_{k} \overline{b_{k}} r^{k-1} e^{-i(k+1) \theta}+\left(c_{m+1} / \lambda_{m+1} \delta\right)\left[\sum_{k=m+1}^{\infty} \lambda_{k} a_{k} r^{k-1} e^{i(k-1) \theta}\right]}{1+\sum_{k=2}^{m} \lambda_{k} a_{k} r^{k-1} e^{i(k-1) \theta}+\sum_{k=2}^{\infty} \mu_{k} \overline{b_{k}} r^{k-1} e^{-i(k+1) \theta}} .
\end{aligned}
$$

So that

$$
\begin{aligned}
& \omega(z) \\
& =\frac{\mathcal{R}\left[\sum_{k=m+1}^{\infty} \lambda_{k} a_{k} r^{k-1} e^{i(k-1) \theta}\right]}{2+2\left(\sum_{k=2}^{m} \lambda_{k} a_{k} r^{k-1} e^{i(k-1) \theta}+\sum_{k=2}^{\infty} \mu_{k} \bar{b}_{k} r^{k-1} e^{-i(k+1) \theta}\right)+\mathcal{R}\left(\sum_{k=m+1}^{\infty} \lambda_{k} a_{k} r^{k-1} e^{i(k-1) \theta}\right)},
\end{aligned}
$$

where $\mathcal{R}$ denotes $\left(c_{m+1} / \lambda_{m+1} \delta\right)$. 
Then

$$
|\omega(z)| \leq \frac{\left(c_{m+1} / \lambda_{m+1} \delta\right)\left[\sum_{k=m+1}^{\infty} \lambda_{k}\left|a_{k}\right|\right]}{2-2\left(\sum_{k=2}^{m} \lambda_{k}\left|a_{k}\right|+\sum_{k=2}^{\infty} \mu_{k}\left|b_{k}\right|\right)-\left(c_{m+1} / \lambda_{m+1} \delta\right)\left(\sum_{k=m+1}^{\infty} \lambda_{k}\left|a_{k}\right|\right)}
$$

This last expression is bounded above by 1 , if and only if

$$
\sum_{k=2}^{m} \lambda_{k}\left|a_{k}\right|+\sum_{k=2}^{\infty} \mu_{k}\left|b_{k}\right|+\frac{c_{m+1}}{\lambda_{m+1} \delta}\left(\sum_{k=m+1}^{\infty} \lambda_{k}\left|a_{k}\right|\right) \leq 1
$$

It suffices to show that L.H.S. of (2.7) is bounded above by $\sum_{k=2}^{\infty}\left(c_{k} / \delta\right)\left|a_{k}\right|+\sum_{k=2}^{\infty}\left(d_{k} / \delta\right)\left|b_{k}\right|$, which is equivalent to

$$
\sum_{k=2}^{m} \frac{c_{k}-\lambda_{k} \delta}{\delta}\left|a_{k}\right|+\sum_{k=2}^{\infty} \frac{d_{k}-\mu_{k} \delta}{\delta}\left|b_{k}\right|+\sum_{k=m+1}^{\infty} \frac{c_{k} \lambda_{m+1}-c_{m+1} \lambda_{k}}{\lambda_{m+1} \delta}\left|a_{k}\right| \geq 0
$$

To see that $f(z)=z+\left(\delta / c_{m+1}\right) z^{m+1}$ gives the sharp result, we observe that for $z=$ $r e^{i \pi / m}$ that

$$
\frac{f(z) * \psi(z)}{f_{m}(z) * \psi(z)}=1+\frac{\lambda_{m+1} \delta}{c_{m+1}} z^{m} \longrightarrow 1-\frac{\lambda_{m+1} \delta}{c_{m+1}}=\frac{c_{m+1}-\lambda_{m+1} \delta}{c_{m+1}}
$$

when $r \rightarrow 1^{-}$.

We next determine bounds for $\operatorname{Re}\left\{\left(f_{m}(z) * \psi(z)\right) /(f(z) * \psi(z))\right\}$.

Theorem 2.2. If $f$ of the form (1.1) with $b_{1}=0$, satisfies the condition (1.4), then

$$
\operatorname{Re}\left\{\frac{f_{m}(z) * \psi(z)}{f(z) * \psi(z)}\right\} \geq \frac{c_{m+1}}{c_{m+1}+\lambda_{m+1} \delta}, \quad(z \in U)
$$

where

$$
c_{k} \geq \begin{cases}\lambda_{k} \delta & \text { if } k=2,3, \ldots, m \\ \frac{\lambda_{k} c_{m+1}}{\lambda_{m+1}} & \text { if } k=m+1, m+2 \ldots\end{cases}
$$

The result (2.10) is sharp with the function given by (2.3). 
Proof. To prove Theorem 2.2, we may write

$$
\begin{aligned}
\frac{1+\omega(z)}{1-\omega(z)} & \\
= & \frac{c_{m+1}+\lambda_{m+1} \delta}{\lambda_{m+1} \delta}\left[\frac{f_{m}(z) * \psi(z)}{f(z) * \psi(z)}-\frac{c_{m+1}}{c_{m+1}+\lambda_{m+1} \delta}\right] \\
& =\frac{1+\sum_{k=2}^{m} \lambda_{k} a_{k} r^{k-1} e^{i(k-1) \theta}+\sum_{k=2}^{\infty} \mu_{k} \overline{b_{k}} r^{k-1} e^{-i(k+1) \theta}-\left(c_{m+1} / \lambda_{m+1} \delta\right)\left[\sum_{k=m+1}^{\infty} \lambda_{k} a_{k} r^{k-1} e^{i(k-1) \theta}\right]}{1+\sum_{k=2}^{\infty} \lambda_{k} a_{k} r^{k-1} e^{i(k-1) \theta}+\sum_{k=2}^{\infty} \mu_{k} \overline{b_{k}} r^{k-1} e^{-i(k+1) \theta}},
\end{aligned}
$$

where

$$
|\omega(z)| \leq \frac{\left(\left(c_{m+1}+\lambda_{m+1} \delta\right) / \lambda_{m+1} \delta\right)\left[\sum_{k=m+1}^{\infty} \lambda_{k}\left|a_{k}\right|\right]}{2-2\left(\sum_{k=2}^{m} \lambda_{k}\left|a_{k}\right|+\sum_{k=2}^{\infty} \mu_{k}\left|b_{k}\right|\right)-\left(\left(c_{m+1}-\lambda_{m+1} \delta\right) / \lambda_{m+1} \delta\right)\left(\sum_{k=m+1}^{\infty} \lambda_{k}\left|a_{k}\right|\right)} \leq 1 .
$$

This last inequality is equivalent to

$$
\sum_{k=2}^{m} \lambda_{k}\left|a_{k}\right|+\sum_{k=2}^{\infty} \mu_{k}\left|b_{k}\right|+\frac{c_{m+1}}{\lambda_{m+1} \delta}\left(\sum_{k=m+1}^{\infty} \lambda_{k}\left|a_{k}\right|\right) \leq 1
$$

Since the L.H.S. of (2.14) is bounded above by $\sum_{k=2}^{\infty}\left(c_{k} / \delta\right)\left|a_{k}\right|+\sum_{k=2}^{\infty}\left(d_{k} / \delta\right)\left|b_{k}\right|$, the proof is evidently complete.

Adopting the same procedure as in Theorems 2.1 and 2.2 and performing simple calculations, we can obtain the sharp lower bounds for the real parts of the following ratios:

$$
\begin{aligned}
\operatorname{Re}\left\{\frac{(f(z) * \psi(z))}{\left(f_{n}(z) * \psi(z)\right)}\right\}, & \operatorname{Re}\left\{\frac{\left(f_{n}(z) * \psi(z)\right)}{(f(z) * \psi(z))}\right\}, \quad \operatorname{Re}\left\{\frac{(f(z) * \psi(z))}{\left(f_{m, n}(z) * \psi(z)\right)}\right\} \\
& \operatorname{Re}\left\{\frac{\left(f_{m, n}(z) * \psi(z)\right)}{(f(z) * \psi(z))}\right\} .
\end{aligned}
$$

The results corresponding to real parts of these ratios are contained in the following Theorems 2.3, 2.4, 2.5, and 2.6.

Theorem 2.3. If $f$ of the form (1.1) with $b_{1}=0$ satisfies the condition (1.4), then

$$
\operatorname{Re}\left\{\frac{f(z) * \psi(z)}{f_{n}(z) * \psi(z)}\right\} \geq \frac{d_{n+1}-\mu_{n+1} \delta}{d_{n+1}}, \quad(z \in U)
$$


where

$$
d_{k} \geq \begin{cases}\mu_{k} \delta & \text { if } k=2,3, \ldots, n \\ \frac{\mu_{k} d_{n+1}}{\mu_{n+1}} & \text { if } k=n+1, n+2 \ldots\end{cases}
$$

The result (2.16) is sharp with the function

$$
f(z)=z+\frac{\delta}{d_{n+1}} \bar{z}^{n+1} .
$$

Theorem 2.4. If $f$ of the form (1.1) with $b_{1}=0$, satisfies the condition (1.4), then

$$
\operatorname{Re}\left\{\frac{f_{n}(z) * \psi(z)}{f(z) * \psi(z)}\right\} \geq \frac{d_{n+1}}{d_{n+1}+\mu_{n+1} \delta}, \quad(z \in U),
$$

where

$$
d_{k} \geq \begin{cases}\mu_{k} \delta & \text { if } k=2,3, \ldots, n \\ \frac{\mu_{k} d_{n+1}}{\mu_{n+1}} & \text { if } k=n+1, n+2 \ldots\end{cases}
$$

The result (2.19) is sharp with the function given by (2.18).

Theorem 2.5. If $f$ of the form (1.1) with $b_{1}=0$, satisfies the condition (1.4), then

(i)

$$
\operatorname{Re}\left\{\frac{f(z) * \psi(z)}{f_{m, n}(z) * \psi(z)}\right\} \geq \frac{c_{m+1}-\lambda_{m+1} \delta}{c_{m+1}}, \quad(z \in U)
$$

where

$$
\begin{gathered}
c_{k} \geq \begin{cases}\lambda_{k} \delta & \text { if } k=2,3, \ldots, m, \\
\frac{\lambda_{k} c_{m+1}}{\lambda_{m+1}} & \text { if } k=m+1, m+2 \ldots,\end{cases} \\
d_{k} \geq \begin{cases}\lambda_{k} \delta & \text { if } k=2,3, \ldots, m, \\
\frac{\lambda_{k} c_{m+1}}{\lambda_{m+1}} & \text { if } k=m+1, m+2 \ldots\end{cases}
\end{gathered}
$$

(ii)

$$
\operatorname{Re}\left\{\frac{f(z) * \psi(z)}{f_{m, n}(z) * \psi(z)}\right\} \geq \frac{d_{n+1}-\mu_{n+1} \delta}{d_{n+1}}, \quad(z \in U)
$$


where

$$
\begin{gathered}
c_{k} \geq \begin{cases}\mu_{k} \delta & \text { if } k=2,3, \ldots, n, \\
\frac{\mu_{k} d_{n+1}}{\mu_{n+1}} & \text { if } k=n+1, n+2 \ldots,\end{cases} \\
d_{k} \geq \begin{cases}\mu_{k} \delta & \text { if } k=2,3, \ldots, n, \\
\frac{\mu_{k} d_{n+1}}{\mu_{n+1}} & \text { if } k=n+1, n+2 \ldots\end{cases}
\end{gathered}
$$

The results (2.21) and (2.23) are sharp with the functions given by (2.3) and (2.18), respectively.

Theorem 2.6. If $f$ of the form (1.1) with $b_{1}=0$, satisfies condition (1.4), then

(i)

$$
\operatorname{Re}\left\{\frac{f_{m, n}(z) * \psi(z)}{f(z) * \psi(z)}\right\} \geq \frac{c_{m+1}}{c_{m+1}+\lambda_{m+1} \delta}, \quad(z \in U)
$$

where

$$
\begin{gathered}
c_{k} \geq \begin{cases}\lambda_{k} \delta & \text { if } k=2,3, \ldots, m, \\
\frac{\lambda_{k} c_{m+1}}{\lambda_{m+1}} & \text { if } k=m+1, m+2 \ldots,\end{cases} \\
d_{k} \geq \begin{cases}\lambda_{k} \delta & \text { if } k=2,3, \ldots, m, \\
\frac{\lambda_{k} c_{m+1}}{\lambda_{m+1}} & \text { if } k=m+1, m+2 \ldots\end{cases}
\end{gathered}
$$

(ii)

$$
\operatorname{Re}\left\{\frac{f_{m, n}(z) * \psi(z)}{f(z) * \psi(z)}\right\} \geq \frac{d_{n+1}}{d_{n+1}+\mu_{n+1} \delta^{\prime}}, \quad(z \in U)
$$

where

$$
\begin{gathered}
c_{k} \geq \begin{cases}\mu_{k} \delta & \text { if } k=2,3, \ldots, m, \\
\frac{\mu_{k} d_{n+1}}{\mu_{n+1}} & \text { if } k=m+1, m+2 \ldots,\end{cases} \\
d_{k} \geq \begin{cases}\mu_{k} \delta & \text { if } k=2,3, \ldots, n, \\
\frac{\mu_{k} d_{n+1}}{\mu_{n+1}} & \text { if } k=n+1, n+2 \ldots\end{cases}
\end{gathered}
$$


The results (2.25) and (2.27) are sharp with the functions given by (2.3) and (2.18) respectively.

\section{Some Consequences and Concluding Remarks}

In this section, we specifically point out the relevances of some of our main results with those results which have appeared recently in literature.

If we put $\psi(z)=(z /(1-z))+\overline{((z /(1-z))-z)}$ and $\psi(z)=\left(z /(1-z)^{2}\right)+$ $\overline{\left(\left(z /(1-z)^{2}\right)-z\right)}$ in Theorems 2.1-2.6, then we obtain the corresponding results of Porwal [28].

Next, if we put $\psi(z)=(z /(1-z))+\overline{((z /(1-z))-z)}, \psi(z)=\left(z /(1-z)^{2}\right)+$ $\overline{\left(\left(z /(1-z)^{2}\right)-z\right)}, c_{k}=k-\alpha, d_{k}=k+\alpha$, and $\delta=1-\alpha$ in Theorems 2.1-2.6, then we obtain the corresponding results of Porwal and Dixit [29].

Again, if we put $g=0$ in Theorems 2.1 and 2.2, then we obtain the corresponding results of Dixit and Porwal [21].

Lastly, if we put $g \equiv 0, \psi(z)=z /(1-z)$, and $\psi(z)=z /(1-z)^{2}$ Theorems 2.1 and 2.2, then we obtain the result of Frasin [6].

We mention below some corollaries giving sharp bounds of the real parts on the ratio of univalent functions to its sequences of partial sums.

By putting $\psi(z)=z /(1-z)$ in Theorem 2.1 for the function $f$ of the form (1.2) with $c_{k}=k-\alpha$ and $\delta=1-\alpha$, then we obtain the following result of Silverman [19], Theorem 1 .

Corollary 3.1. If $f$ of the form (1.2) satisfies the condition (1.5) with $c_{k}=k-\alpha$ and $\delta=1-\alpha$, then

$$
\operatorname{Re}\left\{\frac{f(z)}{f_{m}(z)}\right\} \geq \frac{m}{m+1-\alpha}, \quad z \in U
$$

The result is sharp for every $m$, with the extremal function given by

$$
f(z)=z+\frac{1-\alpha}{m+1-\alpha} z^{m+1}
$$

On the other hand, if we put $\psi(z)=z /(1-z)^{2}$ in Theorem 2.1 for the function $f$ of the form (1.2) with $c_{k}=k-\alpha$ and $\delta=1-\alpha$, then we obtain the following result of Silverman, Theorem 4(i) [19].

Corollary 3.2. If $f$ of the form (1.2) satisfies the condition (1.5) with $c_{k}=k-\alpha$, then for $z \in U$

$$
\operatorname{Re}\left\{\frac{f^{\prime}(z)}{f_{m}^{\prime}(z)}\right\} \geq \frac{\alpha m}{m+1-\alpha}
$$

The result is sharp for every $m$, with the extremal function given by (3.2).

Also, if we put $\psi(z)=z /(1-z)$ in Theorem 2.1 for the function $f$ of the form (1.2) belonging to the class $S_{\phi}\left(c_{k}, \delta\right)$, then we obtain the following result of Frasin [6]. 
Corollary 3.3. If $\in S_{\phi}\left(c_{k}, \delta\right)$, then

$$
\operatorname{Re}\left\{\frac{f(z)}{f_{m}(z)}\right\} \geq \frac{c_{m+1}-\delta}{c_{m+1}}, \quad(z \in U)
$$

where

$$
c_{k} \geq \begin{cases}\delta & \text { if } k=2,3, \ldots, m \\ c_{m+1} & \text { if } k=m+1, m+2, \ldots\end{cases}
$$

The result is sharp for every $m$, with the extremal function given by

$$
f(z)=z+\frac{\delta}{c_{m+1}} z^{m+1}
$$

Next, if we put $\psi(z)=z /(1-z)$ in Theorem 2.1 for the function $f$ of the form (1.2) with $c_{k}=\rho_{k}(\lambda, \gamma, \eta)$ and $\delta=1-\gamma$, then we obtain the following result of Murugusundaramoorthy et al. ([23], Theorem 2.1).

Corollary 3.4. If $f$ of the form (1.2) satisfies the condition (1.5) with $c_{k}=\rho_{k}(\lambda, \gamma, \eta)$ and $\delta=1-\gamma$, then for $z \in U$ :

$$
\operatorname{Re}\left\{\frac{f(z)}{f_{m}(z)}\right\} \geq \frac{\rho_{m+1}(\lambda, \gamma, \eta)-1+\gamma}{\rho_{m+1}(\lambda, \gamma, \eta)}
$$

where

$$
\rho_{k}(\lambda, \gamma, \eta) \geq \begin{cases}1-\gamma & \text { if } k=2,3, \ldots, m \\ \rho_{m+1}(\lambda, \gamma, \eta) & \text { if } k=m+1, m+2 \ldots\end{cases}
$$

The result is sharp for every $m$, with the extremal function given by

$$
f(z)=z+\frac{1-\gamma}{\rho_{m+1}(\lambda, \gamma, \eta)} z^{m+1}
$$

Again, if we set $\psi(z)=(z /(1-z))+(\overline{(z /(1-z))-z})$ in Theorem 2.1, then we obtain the following result of Porwal [28].

Corollary 3.5. If $f$ of the form (1.1) with $b_{1}=0$, satisfies the condition (1.4) with

$$
c_{k} \geq \begin{cases}\delta & \text { if } k=2,3, \ldots, m \\ c_{m+1} & \text { if } k=m+1, m+2 \ldots\end{cases}
$$


then

$$
\operatorname{Re}\left\{\frac{f(z)}{f_{m}(z)}\right\} \geq \frac{c_{m+1}-\delta}{c_{m+1}}, \quad \forall z \in U
$$

The result (3.11) is sharp with the function given by (3.6).

Here we give some open problems for the readers.

In 2004, Owa et al. [24] studied the starlikeness and convexity properties on the partial sums $f_{n}(z)$ and $g_{n}(z)$ of the familiar Koebe function $f(z)=z /(1-z)^{2}$ which is the extremal function for the class $S^{*}$ of starlike functions in the open unit disk $U$ and the function $g(z)=z /(1-z)$ which is the extremal function for the class $K$ of convex functions in the open unit disk $U$, respectively. They also presented some illustrative examples by using Mathematica (Version 4.0). It is interesting to obtain analogues results on harmonic starlikeness and convexity properties of the partial sums of the harmonic Koebe function.

In 2003, Jahangiri et al. [30] studied the construction of sense-preserving, univalent, and close-to-convex harmonic functions by using of the Alexander integral transforms of certain analytic functions (which are starlike or convex of positive order). They construct a function

$$
g(z)=z+\frac{1-\alpha}{k-\alpha} z^{k}+\frac{\alpha}{2}(\bar{z})^{2}+\frac{\alpha(1-\alpha)}{(k+1)(k-\alpha)}(\bar{z})^{k+1},
$$

which is sense-preserving, univalent, and close-to-convex harmonic in $U$, by using the result of Theorem 2 [30] and taking the following function:

$$
f(z)=z+\frac{1-\alpha}{k(k-\alpha)} z^{k}, \quad(k>1 ; 0 \leq \alpha<1) .
$$

It is worthy to note that the function (3.13) is of the form (3.6) with $c_{k}=k(k-\alpha)$ and $\delta=1-\alpha$. Therefore, it is natural to ask that the results of [30] may be generalized for the function of the form (3.6).

\section{Acknowledgment}

The author is thankful to the referee for his valuable comments and observations which helped in improving the paper.

\section{References}

[1] J. Clunie and T. Sheil-Small, "Harmonic univalent functions," Annales Academiae Scientiarum Fennicae. Series A I. Mathematica, vol. 9, pp. 3-25, 1984.

[2] P. Duren, Harmonic Mappings in the Plane, vol. 156, Cambridge University Press, Cambridge, UK, 2004.

[3] O. P. Ahuja, "Planar harmonic univalent and related mappings," Journal of Inequalities in Pure and Applied Mathematics, vol. 6, no. 4, article 122, pp. 1-18, 2005.

[4] S. Ponnusamy and A. Rasila, "Planar harmonic mappings," RMS Mathematics Newsletters, vol. 17, no. 2, pp. 40-57, 2007.

[5] S. Ponnusamy and A. Rasila, "Planar harmonic and quasi-conformal mappings," RMS Mathematics Newsletters, vol. 17, no. 3, pp. 85-101, 2007. 
[6] B. A. Frasin, "Generalization of partial sums of certain analytic and univalent functions," Applied Mathematics Letters, vol. 21, no. 7, pp. 735-741, 2008.

[7] H. Silverman, "Harmonic univalent functions with negative coefficients," Journal of Mathematical Analysis and Applications, vol. 220, no. 1, pp. 283-289, 1998.

[8] H. Silverman and E. M. Silvia, "Subclasses of harmonic univalent functions," New Zealand Journal of Mathematics, vol. 28, no. 2, pp. 275-284, 1999.

[9] J. M. Jahangiri, "Harmonic functions starlike in the unit disk," Journal of Mathematical Analysis and Applications, vol. 235, no. 2, pp. 470-477, 1999.

[10] K. K. Dixit and S. Porwal, "An application of fractional calculus to harmonic univalent functions," Bulletin of the Calcutta Mathematical Society, vol. 102, no. 4, pp. 343-352, 2010.

[11] K. K. Dixit and S. Porwal, "On a subclass of harmonic univalent functions," Journal of Inequalities in Pure and Applied Mathematics, vol. 10, no. 1, article 27, pp. 1-18, 2009.

[12] K. K. Dixit and S. Porwal, "A subclass of harmonic univalent functions with positive coefficients," Tamkang Journal of Mathematics, vol. 41, no. 3, pp. 261-269, 2010.

[13] K. K. Dixit and S. Porwal, "Some properties of harmonic functions defined by convolution," Kyungpook Mathematical Journal, vol. 49, no. 4, pp. 751-761, 2009.

[14] B. A. Frasin, "Comprehensive family of harmonic univalent functions," SUT Journal of Mathematics, vol. 42, no. 1, pp. 145-155, 2006.

[15] M. Öztürk, S. Yalçin, and M. Yamankaradeniz, "Convex subclass of harmonic starlike functions," Applied Mathematics and Computation, vol. 154, no. 2, pp. 449-459, 2004.

[16] S. Porwal, K. K. Dixit, V. Kumar, A. L. Pathak, and P. Dixit, "A new subclass of harmonic univalent functions defined by Dziok-Srivastava Operator," Advances in Theoretical and Applied Mathematics, vol. 5, no. 1, pp. 109-119, 2010.

[17] T. Rosy, B. A. Stephen, K. G. Subramanian, and J. M. Jahangiri, "Goodman-Rønning-type harmonic univalent functions," Kyungpook Mathematical Journal, vol. 41, no. 1, pp. 45-54, 2001.

[18] E. M. Silvia, "On partial sums of convex functions of order $\alpha$, , Houston Journal of Mathematics, vol. 11, no. 3, pp. 397-404, 1985.

[19] H. Silverman, "Partial sums of starlike and convex functions," Journal of Mathematical Analysis and Applications, vol. 209, no. 1, pp. 221-227, 1997.

[20] A. Afaf, Ali Abubaker, and M. Darus, "Partial sums of analytic functions involving generalized ChoKwon-Srivastava operator," International Journal of Open Problems in Complex Analysis, vol. 2, no. 3, pp. 181-188, 2010.

[21] K. K. Dixit and S. Porwal, "A convolution approach on partial sums of certain analytic and univalent functions," Journal of Inequalities in Pure and Applied Mathematics, vol. 10, no. 4, article 101, pp. 1-17, 2009.

[22] B. A. Frasin, "Partial sums of certain analytic and univalent functions," Acta Mathematica, vol. 21, no. 2, pp. 135-145, 2005.

[23] G. Murugusundaramoorthy, K. Uma, and M. Darus, "Partial sums of generalized class of analytic functions involving Hurwitz-Lerch zeta function," Abstract and Applied Analysis, vol. 2011, Article ID 849250, 9 pages, 2011.

[24] S. Owa, H. M. Srivastava, and N. Saito, "Partial sums of certain classes of analytic functions," International Journal of Computer Mathematics, vol. 81, no. 10, pp. 1239-1256, 2004.

[25] S. Porwal and K. K. Dixit, "An application of Salagean derivative on partial sums of certain analytic and univalent functions," Acta Universitatis Apulensis, no. 26, pp. 75-82, 2011.

[26] R. K. Raina and D. Bansal, "Some properties of a new class of analytic functions defined in terms of a Hadamard product," Journal of Inequalities in Pure and Applied Mathematics, vol. 9, no. 1, article 22, pp. 1-20, 2008.

[27] T. Rosy, K. G. Subramanian, and G. Murugusundaramoorthy, "Neighbourhoods and partial sums of starlike functions based on Ruscheweyh derivatives," Journal of Inequalities in Pure and Applied Mathematics, vol. 4, no. 4, article 64, pp. 1-8, 2003.

[28] S. Porwal, "Partial sums of certain harmonic univalent functions," Lobachevskii Journal of Mathematics, vol. 32, no. 4, pp. 366-375, 2011.

[29] S. Porwal and K. K. Dixit, "Partial sums of starlike harmonic univalent functions," Kyungpook Mathematical Journal, vol. 50, no. 3, pp. 433-445, 2010.

[30] J. M. Jahangiri, Y. C. Kim, and H. M. Srivastava, "Construction of a certain class of harmonic closeto-convex functions associated with the Alexander integral transform," Integral Transforms and Special Functions, vol. 14, no. 3, pp. 237-242, 2003. 


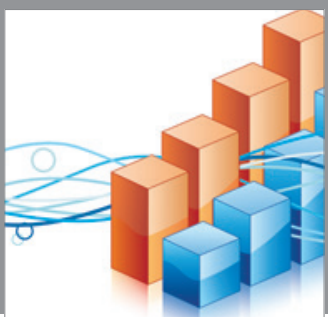

Advances in

Operations Research

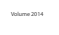

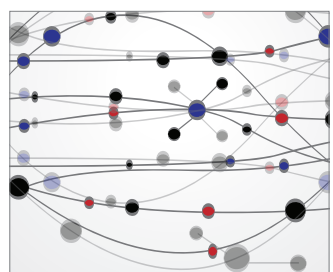

\section{The Scientific} World Journal
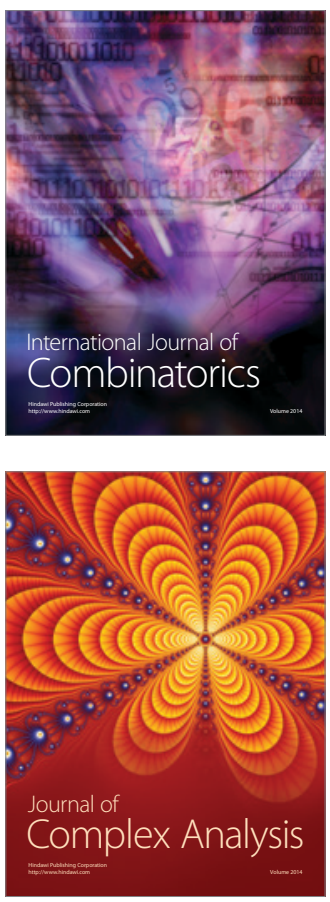

International Journal of

Mathematics and

Mathematical

Sciences
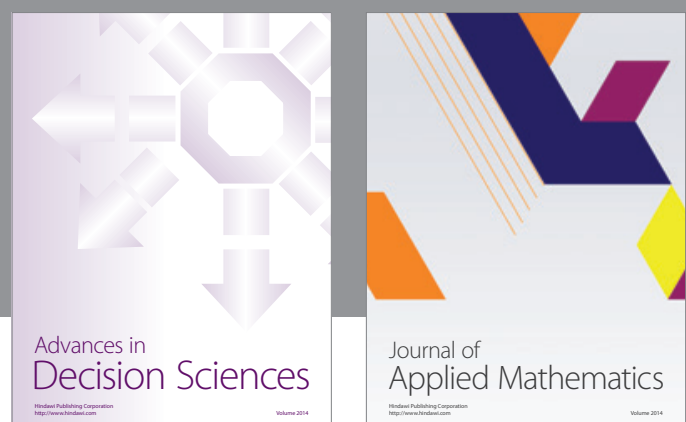

Journal of

Applied Mathematics
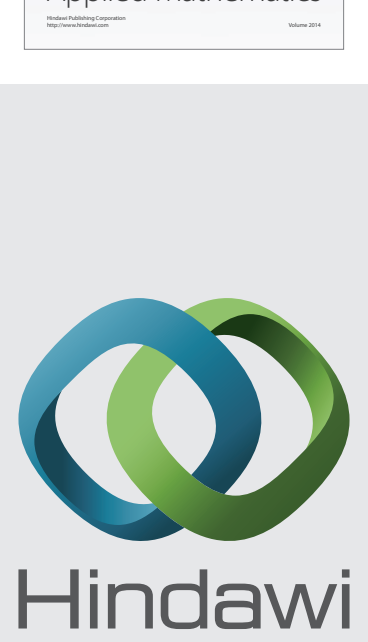

Submit your manuscripts at http://www.hindawi.com
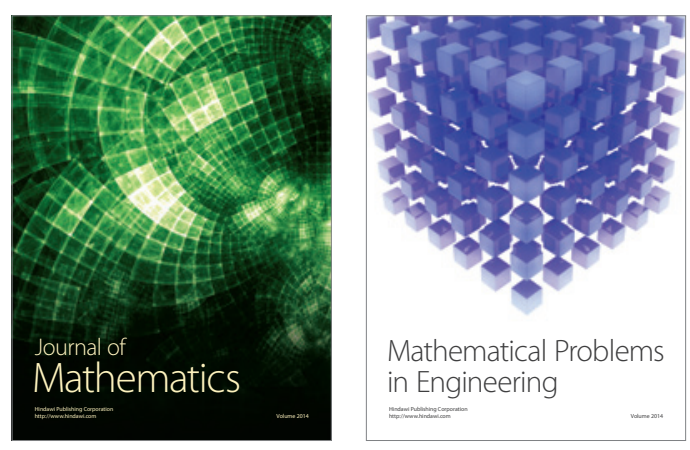

Mathematical Problems in Engineering
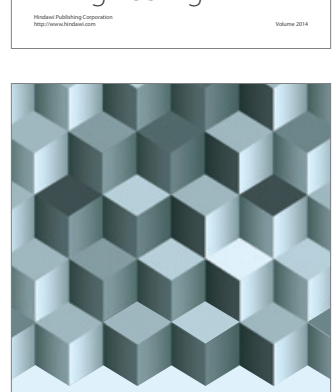

Journal of

Function Spaces
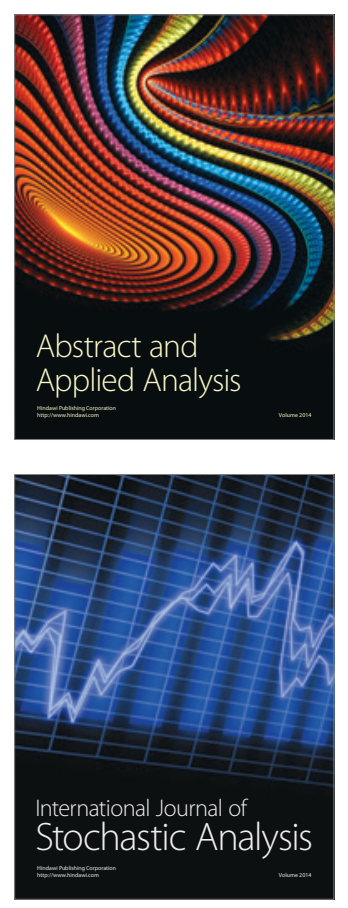

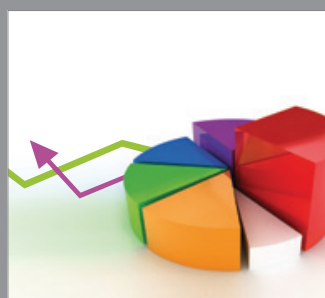

ournal of

Probability and Statistics

Promensencen
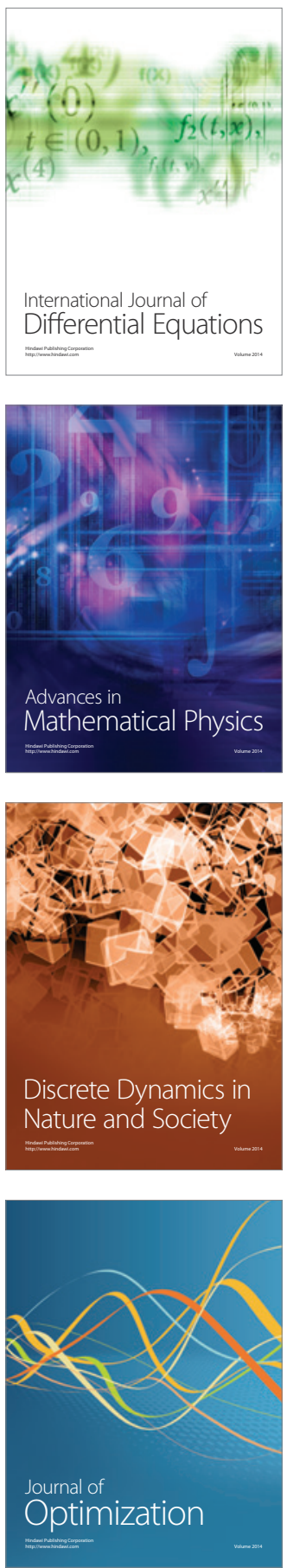